\title{
Making sense of the LHC diboson and diphoton excesses
}

\author{
Suchita Kulkarni*广 \\ Institute of High Energy Physics, Austrian Academy of Sciences, \\ Nikolsdorfergasse 18, 1050 Vienna, Austria \\ E-mail: suchita.kulkarni@oeaw.ac.at
}

\begin{abstract}
Towards the end of Run-1 data taking, an excess in the diboson as well as eejj channel was announced. During the first round of Run-2 data taking, an excess in diphoton channel has been announced. I consider possible explanations of these excesses in two different BSM scenarios. I demonstrate the feasibility of accommodating the diboson and eejj excess in a Left-Right Symmetric Standard Model. For the diphoton excess, I scrutinise a simplified model with scalar resonance coupling to gluons, photons and fermionic dark matter. I illustrate the monojet constraints on such a simplified model scenario and inspect the possibility of reconciling the diphoton excess with dark matter constraints.
\end{abstract}

38th International Conference on High Energy Physics

3-10 August 2016

Chicago, USA

\footnotetext{
*Speaker.

${ }^{\dagger}$ I thank F. F. Deppisch, L. Graf, S. Patra, W. Rodejohann, N. Sahu, U. Sarkar, and D. Barducci, A. Goudelis, D. Sengupta for fruitful collaboartions.
} 


\section{LHC diboson excess}

Both ATLAS and CMS collaborations reported mild excesses in resonance searches in diboson final states at $8 \mathrm{TeV}$ centre-of-mass (CM) energy $p p$ collisions, with integrated luminosities approximately $20 \mathrm{fb}^{-} 1$ c.f. [1] for a detailed summary of the excesses. Along with these the CMS collaboration also reported a mild excess in eejj final state [2]. We analyse the compatibility of the diboson and CMS eejj excesses at the LHC Run-1 data with the Left-Right Symmetric Models (LRSM) containing a D-parity. The LRSM models with D-parity can the light neutrino masses via a linear seesaw mechanism and are an attractive possibility. The LRSM models feature additional right handed gauge bosons $M_{Z_{R}}, M_{W_{R}}$ and heavy right handed neutrinos corresponding to the right handed gauge group $S U(2)_{R}$, with gauge coupling $g_{R}$. The right handed gauge bosons mix with the Standard Model (SM) left handed counterparts with mixing angle $\sin \theta_{L W}^{W}$.

The extended left-right symmetric model (LRSM) is based on the gauge group $S U(2)_{L} \times$ $S U(2)_{R} \times U(1)_{B-L} \times D$ with doublet Higgs and spontaneous $D$-parity breaking, to explain the light neutrino masses via a linear seesaw mechanism. The extension of the LRSM is provided by a singlet fermion $S$. The suppression of neutrino mass arises through the large $D$-parity breaking scale that generates the small scale of lepton number violation, while allowing $S U(2)_{R} \times U(1)_{B-L}$ to be broken at the $\mathrm{TeV}$ scale. We embed the low energy left-right symmetric model in a nonsupersymmetric $S O(10)$ GUT to calculate the difference between the left and right sector gauge couplings $g_{L}$ and $g_{R}$ after $D$-parity breaking.

Remarkably, in order to fit the observed excesses $g_{R} / g_{L} \approx 0.57$ is required and can be achieved with the LRSM models with D-parity respecting the GUT unification at the same time.

In figure 1 (left), we show the obtained fits to various excesses. It can be seen that the diboson and dijet cross sections only depend weakly on the neutrino mass and the LR neutrino mixing. On the other hand, the $e e j j$ cross section delicately depends on these parameters through the branching ratios $\operatorname{Br}\left(W_{R} \rightarrow e N\right)$ and $\operatorname{Br}(N \rightarrow e q q)$. The compatibility of the predicted eejj cross section with the observed excess is shown in figure 1 (right) as a red band in the $\left(M_{N}\right.$-sin $\left.\theta_{L R}^{N}\right)$ parameter plane, while other parameters are fixed to the 'best fit' value. For negligible LR neutrino mixing $\sin \theta_{L R}^{N} \approx 0$, there are two solutions: a large neutrino mass $M_{N} \approx 1.6 \mathrm{TeV}$ and a small neutrino mass $M_{N} \approx 0.3 \mathrm{TeV}$. In between these values, the excess could be explained through the inclusion of large LR neutrino mixing up to $\sin \theta_{L R}^{N} \approx 2 \times 10^{-3}$, of the same order as the suggested LR $W$ boson mixing. We thus demonstrate that by interpreting the excesses in such a simplified LRSM would have profound impact on the properties of the heavy neutrino.

\section{LHC diphoton excess}

In this section we take a look at the $750 \mathrm{GeV}$ diphoton excess observed by ATLAS and CMS collaborations in the early $13 \mathrm{TeV}$ centre-of-mass (CM) energy pp collisions. Although it has a statistically mild significance, the excess was reported to be compatible with a particle as broad as $45 \mathrm{GeV}$ with substantial cross-section in the diphton final state.

One interesting way through which a broad resonance can be explained is by invoking decays into dark matter (DM). Such a model can be tested via several ways including the compatibility with measured relic density, DM direct and indirect detection searches and finally LHC monojet 

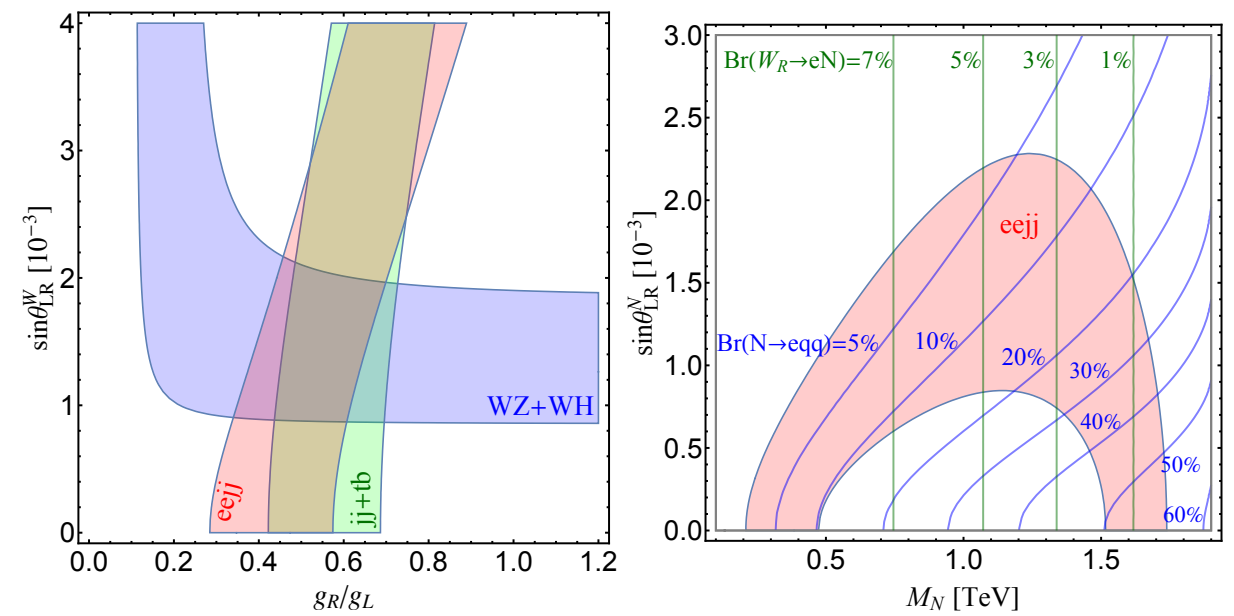

Figure 1: Fitting the diboson $W Z, W H$ (blue band), dijet $j j+t b$ (green band) and $e e j j$ (red band) excesses in the $\left(g_{R} / g_{L}-\sin \theta_{L R}^{W}\right)$ parameter plane. The other parameters are chosen as $M_{W_{R}}=1.9 \mathrm{TeV}, M_{N}=1.6 \mathrm{TeV}$ and $\sin \theta_{L R}^{N}=0$. (left) Fitting the $e e j j$ (red band) excess in the $\left(M_{N}-\sin \theta_{L R}^{N}\right)$ parameter plane. The other parameters are chosen as $M_{W_{R}}=1.9 \mathrm{TeV}, g_{R} / g_{L}=0.57$ and $\sin \theta_{L R}^{W}=1.5 \times 10^{-3}$. The vertical green lines denote contours of constant $\operatorname{Br}\left(W_{R} \rightarrow e N_{R}\right)$ and the diagonal blue lines of constant $\operatorname{Br}\left(N_{R} \rightarrow e q q\right)$ as denoted. (right)

searches. We make an effort to put some of these pieces together in a systematic manner [3]. We recast a supersymmetry (SUSY) monojet search to obtain constraints on the parameter space of the considered model and show their interplay with the diphoton resonance production cross section, its decay width into invisible final states, $13 \mathrm{TeV}$ dijet cross section predictions as well as with cosmological considerations on DM.

More concretely, we consider a scalar resonance coupling to the Standard Model (SM) gauge bosons and Majorana fermion DM $(\psi)$. We neglect all potential couplings of $s$ to SM fermions (which, for a singlet $s$, can also only arise through higher-dimensional operators) as well as to the $125 \mathrm{GeV}$ Higgs boson (which are allowed at tree-level).

$$
\begin{aligned}
\mathscr{L}_{\mathrm{NP}, \mathrm{CPE}} & =\frac{1}{2}\left(\partial_{\mu} s\right)^{2}-\frac{\mu_{s}^{2}}{2} s^{2}+\frac{1}{2} \bar{\psi}\left(i \not \supset-m_{\psi}\right) \psi-\frac{y_{\psi}}{2} s \bar{\psi} \psi \\
& -\frac{g_{1}^{2}}{4 \pi} \frac{1}{4 \Lambda_{1}} s B_{\mu \nu} B^{\mu \nu}-\frac{g_{2}^{2}}{4 \pi} \frac{1}{4 \Lambda_{2}} s W_{\mu \nu} W^{\mu \nu}-\frac{g_{3}^{2}}{4 \pi} \frac{1}{4 \Lambda_{3}} s G_{\mu \nu} G^{\mu \nu}
\end{aligned}
$$

where $B_{\mu \nu}, W_{\mu \nu}$ and $G_{\mu \nu}$ are the $U(1)_{Y}, S U(2)_{L}$ and $S U(3)_{c}$ field strength tensors respectively and $g_{1,2,3}$ are the corresponding SM coupling constants. $\Lambda_{1,2,3}$ are independent suppression mass scales. The Lagrangian (2.1) actually corresponds to the case where $s$ is even under the chargeparity $(C P)$ symmetry. A similar Lagrangian for CP-odd case can be written down. The collider phenomenology aspects of $s$ we will focus on depend only mildly on its $C P$ nature, unlike the DM properties of $\psi$.

In figure 2, we overlay several constraints obtained in this model for fixed value of DM mass $\left(m_{\psi}\right)$ and suppression scale $\Lambda_{1,2}$. Leaving monojet constraints aside for the moment, we see that in the $m_{\psi}=250 \mathrm{GeV}$ case the requirements for a a substantial diphoton cross section and a large 


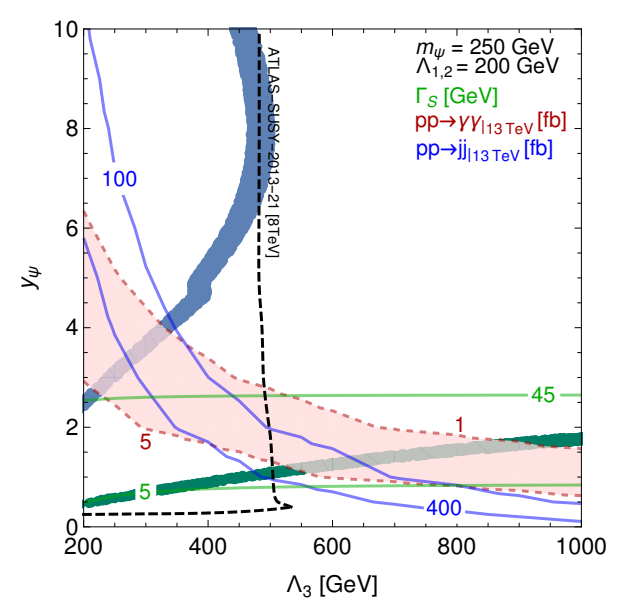

Figure 2: Predictions for $p p \rightarrow s \rightarrow \gamma \gamma$ (red band) and $p p \rightarrow s \rightarrow j j$ (blue contours) cross sections at $\sqrt{s}=13$ $\mathrm{TeV}$, overlaid with $8 \mathrm{TeV}$ monojet constraints (black line) and the width of the resonance $s$ (green contours). The mass of the invisible fermion $\psi$ is fixed at $m_{\psi}=250 \mathrm{GeV}$ and $\Lambda_{1,2}=200 \mathrm{GeV}$. Monojet constraints are derived at $95 \%$ C.L. The blue (green) band shows regions of parameter space compatible with the observed DM density for a scalar (pseudoscalar) mediator.

resonance width $\Gamma_{s}>20 \mathrm{GeV}$ can be reconciled in substantial parts of the parameter space. The relic abundance constraint for $\psi$ significantly reduces the available parameter space, although it is still possible to accommodate all three requirements assuming a CP-odd scalar. The imposition of the monojet constraints has an important impact on the parameter space, excluding $\Lambda_{3}$ values below $\sim 500 \mathrm{GeV}$ regardless of the value of $y_{\psi}$, unless $y_{\psi} \leq 0.25$. A detailed discussion on the effect of changing $m_{\psi}$ or $\Lambda_{1,2,3}$ can be found in [3].

\section{Conclusions}

The transient nature of LHC excesses so far observed demands us to be patient. However, it is an educating exercise to make sense of the early hints of the excesses. Although both the diphoton and the diboson excesses are not confirmed by the subsequent searches, many new theories were thought to explain them. It only shows the landscape of theoretical scenarios so far unexplored. With an open mind and hope, we should look forward to new developments in theory and results from the LHC.

\section{References}

[1] F. F. Deppisch, L. Graf, S. Kulkarni, S. Patra, W. Rodejohann, N. Sahu and U. Sarkar, Phys. Rev. D 93 (2016) no.1, 013011 doi:10.1103/PhysRevD.93.013011 [arXiv:1508.05940 [hep-ph]].

[2] V. Khachatryan et al. [CMS Collaboration], Eur. Phys. J. C 74 (2014) no.11, 3149 doi:10.1140/epjc/s10052-014-3149-z [arXiv:1407.3683 [hep-ex]].

[3] D. Barducci, A. Goudelis, S. Kulkarni and D. Sengupta, JHEP 1605 (2016) 154 doi:10.1007/JHEP05(2016)154 [arXiv:1512.06842 [hep-ph]]. 